\title{
Management and outcome of suspected and confirmed COVID-19 (SARS-CoV-2) vaccine hypersensitivity.
}

Margitta Worm ${ }^{1}$, A. Alexiou ${ }^{1}$, Andrea Bauer ${ }^{2}$, Regina Treudler ${ }^{3}$, Gerda Wurpts ${ }^{4}$, Heinrich Dickel $^{5}$, Timo Buhl ${ }^{6}$, Sabine Müller ${ }^{7}$, Andreas Jung ${ }^{8}$, R. Brehler ${ }^{9}$, Joachim Fluhr ${ }^{10}$, Ludger Klimek $^{11}$, N. Mülleneisen ${ }^{12}$, W. Pfützner ${ }^{13}$, Ulrike Raap ${ }^{14}$, Stefani Röseler ${ }^{15}$, S. Schuh ${ }^{16}$, H. Timmermann $^{17}$, Guido Heine ${ }^{18}$, Bettina Wedi ${ }^{19}$, and Knut Brockow ${ }^{20}$

${ }^{1}$ Charite Universitatsmedizin Klinik fur Dermatologie Venerologie und Allergologie

${ }^{2}$ Klinik und Poliklinik für Dermatologie Comprehensive Allergy Center (CAC) Universitätsklinikum Carl Gustav Carus an der Technischen Universität Dresden

${ }^{3}$ Universitatsklinikum Leipzig Klinik und Poliklinik fur Dermatologie Venerologie und Allergologie

${ }^{4}$ Klinik für Dermatologie und Allergologie Aachener Comprehensive Allergy Center (ACAC) Universitätsklinik der RWTH Aachen

${ }^{5}$ Abteilung für Allergologie Berufs- und Umweltdermatologie Klinik für Dermatologie Venerologie und Allergologie St Josef-Hospital Ruhr-Universität Bochum

${ }^{6}$ Klinik für Dermatologie Venerologie und Allergologie Universitätsmedizin Göttingen Georg-August-Universität

${ }^{7}$ Klinik für Dermatologie und Allergologie Universitätsklinikum Freiburg

${ }^{8}$ Klinik für Dermatologie Venerologie und Allergologie Universitätsklinikum Gießen

${ }^{9}$ Ambulanz für Allergologie Berufsdermatologie und Umweltmedizin Universitätsklinikum Münster

${ }^{10}$ Comprehensive Allergy Centre Charité CACC Campus Benjamin Franklin Charité Universitätsmedizin Berlin

${ }^{11}$ Allergiezentrum Wiesbaden/Rhein-Main Zentrum für Rhinologie und Allergologie

${ }^{12}$ Asthma- und Allergiezentrum Leverkusen

${ }^{13}$ Allergiezentrum Hessen Klinik für Dermatologie und Allergologie Universitätsklinikum Marburg

${ }^{14}$ Allergiezentrum Oldenburg Universitätsklinik für Dermatologie und Allergologie Klinikum Oldenburg AöR

${ }^{15}$ Klinik für Pneumologie Allergologie Schlaf- und Beatmungsmedizin Krankenhaus der Augustinerinnen Köln Kooperationspartner Aachener Comprehensive Allergy Center (ACAC)

${ }^{16}$ Klinik für Dermatologie und Allergologie Universitätsklinikum Augsburg - am Standort Medizincampus Süd Augsburg

${ }^{17}$ Schwerpunktpraxis Colonaden Hamburg

${ }^{18}$ Klinik für Dermatologie Venerologie und Allergologie Universitätsklinikum Schleswig-Holstein Campus Kiel Kiel

${ }^{19}$ Klinik für Dermatologie Allergologie und Venerologie Comprehensive Allergy Center (CAC) Medizinische Hochschule Hannover 


\title{
${ }^{20}$ Klinik und Poliklinik für Dermatologie und Allergologie am Biederstein Technische Universität München
}

October 20, 2021

\begin{abstract}
Systemic allergic reactions to vaccines are very rare. In this study we assessed the management and outcome suspected SARSCoV-2 vaccine hypersensitivity. We present data of 219 individuals, who experienced symptoms suspicious for an allergic reaction after the first $(n=214)$ or the second vaccination $(n=5) .195$ reactions occurred after the first application of mRNAbased vaccines (157 Comirnatyß), and 38 Spikevaxß) and eighteen reactions were reported after first application of a vector vaccine (Vaxzevria@). Of these 162 experienced immediate symptoms. Skin symptoms occurred in 91 cases. The most frequent cutaneous symptom was angioedema $(n=45)$, followed by generalized urticaria $(n=36)$ and generalized erythema/flush $(n=20)$. 70 patients had cardiovascular symptoms, 45 showed respiratory symptoms and gastrointestinal symptoms were recorded in 14 patients. The allergological assessment of 334 individuals (219 with reactions after COVID vaccination and 115 with a history of vaccine related reactions) showed in $17 \%$ a suspicion of sensitization against the SARS-CoV-2 vaccine and/or their ingredients defined as one positive skin test and/or BAT. The majority of the SPT/IDT with the vaccines were negative. Of the 214 patients with suspected allergic symptoms after the first vaccination, 67/67 patients tolerated the re-vaccination. In this study, 334 individuals of a cohort resembling $>2000$ persons presenting for an allergy workup regarding SARS-CoV-2 vaccination only 45 were diagnosed in concordance with the anaphylaxis definition of the Brighton collaboration with anaphylactic immediate hypersensitivity reaction after SARS-CoV-2 vaccination. Identifiable characteristics of these patients with suspected, but also diagnosed SARS-CoV-2 vaccine hypersensitivity were female gender and the symptom angioedema. Overall, IgE-mediated hypersensitivity towards SARS-CoV-2 vaccines is extremely low and not increased in comparison to the reported hypersensitivity for other vaccines.
\end{abstract}

\section{Introduction}

In December 2021, the vaccination campaigns against the COVID-19 pandemic were initiated on an international level. Soon after authorization, reports on severe allergic reactions and anaphylaxis in the context of SARS-CoV-2 vaccination raised the alertness particularly in patients with a history of previous severe allergic reactions, atopic diseases and other allergic manifestations [1]. Until now four SARS-CoV-2 vaccines have been licensed in Germany: the mRNA vaccines Comirnaty@ (BioNTech Manufacturing GmbH) and Spikevax@ (MODERNA BIOTECH SPAIN, S.L.) and the vector vaccines Vaxzevria@ (AstraZeneca AB) and COVID-19 vaccine Janssen@ (Johnson \& Johnson).

Vaccine related side effects may be caused by the excipients of the mRNA and vector vaccines, polyethylene glycol (PEG)/ polysorbate 80 (PS80) [2, 3]. Based on previous experience systemic allergic reactions to vaccines are very rare and range between $1-5$ cases per 1 one million applications [4]. Regarding the Pfizer/ BioNTech BNT162B2 (Comirnaty@) SARS-CoV-2 Vaccine, by January 20214.7 cases of allergic reactions occurred per 1 one million applications in the US [5].

Herewith we established a multicenter protocol for individuals with suspected SARS-CoV-2 vaccination hypersensitivity within German Comprehensive Allergy Centers (CAC) (Berlin, Giessen, Göttingen, Hannover, Leipzig, Marburg, Dresden, Munich, Aachen, Oldenburg) and other large allergy centers in Germany (Augsburg, Bochum, Kiel, Köln, Freiburg, Hamburg, Leverkusen, Wiesbaden) in order to identify IgE-mediated hypersensitivity against SARS-CoV-2 vaccines as well as their excipients and to evaluate the frequency and characteristics of patients with respective hypersensitivities.

\section{Methods}

\section{Patients and procedures}

Adult patients were included in this data analysis, if they met at least one of the following inclusion criteria: 
previous hypersensitivity reactions to vaccines or to medical products containing PEG or PS80, previous idiopathic anaphylaxis, and increased risk of developing anaphylaxis (e.g., because of mastocytosis). Patients were seen by an allergist and after taking a detailed medical history, the allergological workup was performed, including skin prick tests (SPT) and in selected cases also intradermal tests (IDT), as well as detection of total IgE and tryptase.

Since PEG and PS80 are suspected compounds to elicit hypersensitivity reactions, we included PEG2000, PEG6000 and PS80 in the test panel (SPT: 1\%, 10\%; IDT: 0.01\%, 0.1\%, dilution agent: sterile aqua). PEG and PS80 were purchased at Carl Roth (Karlsruhe, Germany). The powder of the substances was prepared by the local pharmacies for further testing. In some centers, Trometamol and 1,2-Distearoyl-sn-glycero-3phosphocholine (DSCP) were applied for SPT at 1\% and 10\% (dilution agent: sterile aqua), these data will not be presented in this analysis. If available, the vaccines were tested undiluted in SPT and at 1\% and 10\% in IDT (dilution agent: sterile aqua). In individual cases, basophil activation tests (BAT) were performed with different compounds (i.e. PEG 2000, 1,2-Dimyristoyl-rac-glycero-3-methoxypolyethylene glycol-2000 1,2-Dimyristoyl-rac-glycero-3-methoxypolyethylene glycol-2000 1,2-Dimyristoyl-rac-glycero-3-methoxy-PEG 2000) in selected centers utilizing the Bühlmann Flow-CAST test (Bühlmann Laboratories AG; Basel, Switzerland) and the results were evaluated according to the company's recommendations. Some centers followed internal protocols for the basophil activation tests, in which vaccine excipients were used at different concentrations for stimulation. The assessment of the clinical data for research proposes was approved by the ethics committee at the Charité (EA1/049/21) as the initiating center. All subjects gave written informed consent.

\section{Results}

\section{Study cohort}

In total, 334 of $>2000$ individuals contacting specialized allergy centers because a self- and-or clinically suspected (Fig.1) immediate-type SARS-CoV-2 vaccine hypersensitivity were investigated (Fig. 2). The median age of this cohort was 49 years (19-91), and 291/334 were female (87.1\%). Medical history data were available for $151 / 334$ patients. The history of the patients included anaphylaxis in most cases to previous vaccination or drugs known or suspected to contain PEG or PS80. Few patients reported on immediate reactions after the use of cosmetics, were sensitized to multiple compounds or showed systemic reactions to contrast media (Table 1).

\section{Reported cases in allergy centers}

Of the 334,214 experienced any symptom in line with a possible allergic reaction after receiving a SARS-CoV2 vaccination and five patients experienced symptoms in line with a possible allergic reaction after receiving the second vaccine dose. Out of these, 195 reactions occurred after the first application of an mRNA-based vaccine (157 Comirnaty $\Omega$, and 38 Spikevax $囚$ ) and eighteen reactions were reported from patients after

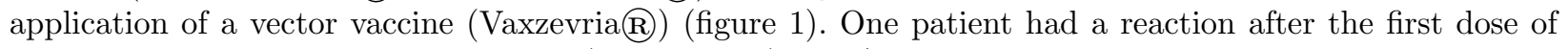
an unknown SARS-CoV-2 vaccination (Sup.Table 3). 115/334 included individuals had a positive allergy history and presented for the assessment of the risk to develop hypersensitivity reactions during SARS$\mathrm{CoV}-2$ vaccination. Of note, we only included those patients who underwent allergy testing in this analysis. Hundreds of patients more presented to the allergy centers with alleged SARS-CoV-2 vaccination reactions, but after taking a detailed history their symptoms were not considered to be allergic and further allergy work-up was omitted. Figure 2 shows a workflow of our study.

Symptoms in the context of SARS-CoV-2 vaccination

219 patients experienced any symptom suggestive for an allergic reaction either after the first dose of vaccination $(n=214)$ or after the second dose of vaccination $(n=5) .162$ experienced immediate symptoms in line with a possible allergic reaction and 55 patients showed other adverse reactions or delayed reactions (maculopapular rash $\mathrm{n}=12$, large local reaction at injection site $\mathrm{n}=8$ ).

Skin symptoms occurred in the majority of the patients $(n=91)$. The most frequent cutaneous symptom 
was angioedema $(n=45)$, followed by generalized urticaria $(n=36)$ and generalized erythema/flush $(n=20)$. 70 patients had cardiovascular symptoms; respiratory symptoms were also frequent $(n=45)$ with dyspnea reported in 45 patients. Gastrointestinal symptoms were recorded in 14 patients only. The majority of the symptoms occurred within less than one hour after vaccination, however in a few cases the symptoms appeared within 24 hours after vaccination ( 7 cases with reported symptoms in line with a possible allergic reaction after 24 hours after vaccination and 4 cases with reported symptoms in line with a possible allergic reaction within 6 to 24 hours after vaccination).

In table 2, patients who experienced immediate symptoms in line with a possible allergic reaction after SARS-CoV-2 vaccination $(\mathrm{n}=162)$ are summarized according to the Brighton levels [6]. Only 45 patients met the criteria of anaphylaxis according to the Brighton criteria (level 1 corresponds to the highest, levels 2 and 3 to lower degrees of diagnostic certainty).

\section{Rate of SARS-CoV-2 vaccine hypersensitivity}

The allergy workup revealed from a total of 334 patients in 57 cases (17\%) a clinical suspicion of type 1 sensitization against the SARS-CoV-vaccine and/or the excipients defined as one positive skin test and/or basophil activation test. The majority of the SPT and IDT with the vaccines were negative. Two patients had a systemic reaction after the IDT (one for Comirnaty@ and one for Spikevax@). Baseline tryptase was not elevated in any of the patients except one who had a known mastocytosis, the median value of baseline tryptase of a total of 113 measured cases was $4.49 \mu \mathrm{g} / \mathrm{ml}$. The BAT was positive in seventeen cases (Table $3)$.

As mentioned above 45 patients met the criteria of anaphylaxis according to the Brighton criteria (level 1 corresponds to the highest, levels 2 and 3 to lower degrees of diagnostic certainty) [4]. The patients were mostly females (42/45 cases, 93.3\%). Of these in 11 cases, at least one diagnostic test was positive (Table 4). The data revealed that 3 patients had a positive BAT for the suspected SARS-CoV-2 vaccine and 4 patients were positive to PEG2000 in the IDT.

\section{Tolerability of re-vaccination}

Of the 214 patients showing any symptom in line with a possible allergic reaction after the first vaccination, 71 patients did not show any immediate allergic symptoms after re-vaccination with the identical $(\mathrm{n}=57)$ or a non-identical SARS-CoV-2 vaccine $(\mathrm{n}=13)$ and for one patient the type of the second vaccine is unknown. Six further patients showed similar tolerability to re-vaccination and one patient had urticarial after the re-vaccination. In some of the above mentioned cases, the second dose was given fractionated $(n=9)$, in other cases, the vaccination was given as a whole dose with extended emergency preparedness and some patients received premedication before vaccination $(n=3)$ (antihistamines and oral corticosteroids).

Finally, two cases were identified for which the vaccination with the approved SARS-CoV-2 vaccinations is contraindicated. Five patients denied revaccination, one patient denied completely the vaccination and one patient died (due to other medical reasons) and could not be revaccinated. For one patient the re-vaccination was not applicable, due to previous COVID-19 Infection.

The allergy workup revealed from a total of 334 patients in 57 cases (17\%) a suspicion of type 1 sensitization against the SARS-CoV-vaccine and/or the excipients defined as one positive skin test and/or basophil activation test. A re-vaccination with any available SARS-CoV-2 vaccine of patients from this group was contraindicated in one case only, due to SPT positivity to several vaccines. $27 / 57$ patients were successfully re-vaccinated (26 with the identical vaccine). Three patients denied completely the vaccination, in one patient of these the re-vaccination was not necessary, due to previous COVID-19 infection and one patient died (due to other medical reasons).

\section{Paul-Ehrlich-Institute reported cases}

From the beginning of the vaccination campaign in Germany on $27^{\text {th }}$ of December 2020 through $31^{\text {st }}$ of July $2021, n=131,671$ suspected cases of any kind of adverse reactions or vaccination complications have been 
reported to the Paul-Ehrlich-Institut, thereof $n=390$ cases of suspected anaphylaxis [7], in the context of vaccination with the mRNA vaccines Comirnaty@ (BioNTech Manufacturing GmbH) and Spikevax@ (MODERNA BIOTECH SPAIN, S.L.) as well as the vector vaccines Vaxzevria $($ ) (AstraZeneca AB) and COVID19 vaccine Janssen $\left(\mathrm{B}\right.$. By August $1^{\text {st }} 2021$, according to data from the Robert Koch Institute 92,376,787 vaccinations had been administered, including 68,962,481 vaccinations with Comirnaty@, 8,506,260 vaccinations with Spikevax@ $\mathrm{B}, 12,491,937$ vaccinations with Vaxzevria $($, and 2,416,109 vaccinations with COVID-19 vaccine Janssen $($.

Concerning reported allergic reactions, the 390 cases with suspected anaphylactic reactions reported until $31^{\text {st }}$ of July 2021, were assessed by the Paul-Ehrlich-Institute as Brighton Collaboration (BC) levels 1-4 (level 1 corresponds to the highest, levels 2 and 3 to lower degrees of diagnostic certainty, and level 4 are reports of suspected anaphylaxis with incomplete information on clinical symptoms) [4]. The number of reported cases of suspected anaphylaxis are specified according to vaccine and applied dose in table 5.

Based on these data, a suspected hypersensitivity to SARS-CoV-2 vaccines results to be 2.7 cases of allergic reactions per 1 one million vaccine applications (considering the Brighton collaboration criteria 1-3). These data are presented in spontaneous reporting and over- or underreporting may be possible.

\section{Discussion}

Confirmed anaphylaxis towards an ingredient of a vaccine is extremely rare and may reach an estimated rate of 1-2 cases per million vaccinations in Germany [8-10]. After starting the world-wide vaccination program against COVID-19, an increased reaction rate for SARS-CoV-2 vaccines has been observed [3] with hypersensitivity against PEG being suspected to be causal [1]. However, only in exceptional cases evidence for PEG as the culprit could be substantiated [11]. Thus, the association between PEG allergy and anaphylaxis to SARS-CoV-2 vaccines remains uncertain.

So far drug and/or vaccine induced hypersensitivity reactions can be caused either IgE-dependent, via a G-protein signalling pathway (MRG-PX2) or through activation of the complement system [12]. Whether PEGs or other vaccine excipients are capable to induce a hypersensitivity reaction besides the IgE dependent pathway is currently not known.

In this multicenter data assessment, out of 334 individuals with suspected hypersensitivity to SARS-CoV2 vaccines and presenting for an allergy workup only 45 were diagnosed with immediate hypersensitivity reactions after vaccination, according to Brighton criteria. As reported previously, patients were mostly females [13]. The overall analyses of the symptom profiles of these patients revealed angioedema to be more common in this patient group, even more frequent than urticaria. This finding is interesting as acquired angioedema shows a predominance in female middle-aged patients as well and may indicate a role of sex hormones for the development of the observed hypersensitivity reactions. In addition previous studies have shown that females experience allergic symptoms more often, e. g. in food allergies, although being less frequently sensitized [14].

The allergy workup in our cohort showed very few positive skin test reactions and almost all of them appeared only in IDT. As unspecific positive IDT reactions are not uncommon in testing drugs, particularly vaccines, positive results have to be interpreted with great caution [9]. Nevertheless, negative skin tests in a large proportion of patients applying recommended test concentrations indicate that the test conditions have a high specificity $>95 \%$ and are suited to impede concerns of doctors and patients against allergy towards SARS-CoV-2 vaccines.

The basophil activation test appears to be non-irritant in the concentrations tested, but only provided additional information in exceptional patients and needs further validation. In the vast majority of patients, after allergy testing, an allergic reaction to PEG, PS80, DSCP and trometamol was ruled out and further vaccination recommended. Even in those few patients with positive reactions in the IDT, unspecific and irritant reactions cannot be finally ruled out, as patients were advised to receive a vaccine not containing the ingredient leading to a positive skin test reaction. 
Tolerability of the second vaccine dose shown by us and by other groups [15] suggest that re-vaccination is safe in the vast majority of these patients. As some symptoms concerning the respiratory tract, circulatory or gastrointestinal system are subjective, these might be an expression of anxiety rather than an allergic or other adverse organ reaction or may be triggered via vasovagal activation.

Thus, we propose that patients reporting systemic reactions after SARS-CoV-2 vaccination should be carefully evaluated for differential diagnosis, e.g., vasovagal, or stress-triggered reactions. If possible, patients should be evaluated for an increased serum tryptase 2-4 hours after the reaction to gather further evidence for an allergic reaction, and a thorough allergy workup should follow. Here, we propose a SPT and - if negative - in selected cases IDT with both the SARS-CoV-2 vaccines and hypersensitivity eliciting ingredients. Recent data from the literature indicate that a SPT with 50\% PEG 20,000 may be useful as a screening test for PEG allergy when lower MW PEGs test are negative, whereas IDT with PEG requires confirmation regarding safety and validity [16].

Overall, IgE-mediated hypersensitivity towards SARS-CoV-2 vaccines is extremely low and not increased in comparison to the reported hypersensitivity rates for other vaccines. However, the tremendous amount of patients seeking allergological advice regarding the tolerability of COVID-19 vaccination points to the need of appropriate information campaigns in the general population in order to facilitate high vaccination rates.

References

1. Worm, M., et al., [Covid-19 vaccination and risk of anaphylaxis - Recommendations for practical management]. MMW Fortschr Med, 2021. 163 (1): p. 48-51.

2. Garvey, L.H. and S. Nasser, Anaphylaxis to the first COVID-19 vaccine: is polyethylene glycol (PEG) the culprit? Br J Anaesth, 2021.126 (3): p. e106-e108.

3. Kraft, M., et al., Anaphylaxis to vaccination and polyethylene glycol: a perspective from the European Anaphylaxis Registry. J Eur Acad Dermatol Venereol, 2021.

4. Dreskin, S.C., et al., International Consensus (ICON): allergic reactions to vaccines. World Allergy Organ J, 2016. 9 (1): p. 32.

5. Shimabukuro, T.T., M. Cole, and J.R. Su, Reports of Anaphylaxis After Receipt of mRNA COVID-19 Vaccines in the US-December 14, 2020-January 18, 2021. Jama, 2021. 325 (11): p. 1101-1102.

6. Rüggeberg, J.U., et al., Anaphylaxis: case definition and guidelines for data collection, analysis, and presentation of immunization safety data. Vaccine, 2007. 25 (31): p. 5675-84.

7. Paul-Ehrlich-Institut, Bericht über Verdachtsfälle von Nebenwirkungen und Impfkomplikationen nach Impfung zum Schutz vor COVID-19 (Berichtszeitraum 27.12. bis 31.07.2021) . 2021.

8. Lange, L., Allergene in Impfstoffen. Allergologie, 201639 (8): p. 357-361.

9. Nilsson, L., et al., Vaccination and allergy: EAACI position paper, practical aspects. Pediatr Allergy Immunol, 2017.28 (7): p. 628-640.

10. Stone, C.A., Jr., et al., Immune-mediated adverse reactions to vaccines. Br J Clin Pharmacol, 2019. 85 (12): p. 2694-2706.

11. Sellaturay, P., et al., Polyethylene glycol (PEG) is a cause of anaphylaxis to the Pfizer/BioNTech mRNA COVID-19 vaccine. Clin Exp Allergy, 2021. 51 (6): p. 861-863.

12. Worm, M., et al., Drug-induced anaphylaxis-elicitors, mechanisms and diagnosis. Allergo Journal International, 2019.28 .

13. Krantz, M.S., et al., Safety Evaluation of the Second Dose of Messenger RNA COVID-19 Vaccines in Patients With Immediate Reactions to the First Dose. JAMA Intern Med, 2021. 
14. Soost, S., et al., Risk factors of adverse reactions to food in German adults. Clin Exp Allergy, 2009. 39 (7): p. 1036-44.

15. Rasmussen, T.H., et al., Patients with suspected allergic reactions to COVID-19 vaccines can be safely revaccinated after diagnostic work-up. Clinical and Translational Allergy, 2021.11 (5): p. e12044.

16. Bruusgaard-Mouritsen, M.A., et al., Optimizing investigation of suspected allergy to polyethylene glycols. J Allergy Clin Immunol, 2021.

Tables and figures

Table 1: Medical history of the patients, $n=151^{*}$

\begin{tabular}{ll}
\hline Medical History & Number of patients \\
\hline anaphylaxis (not further specified) & 8 \\
drug allergy & 53 \\
confirmed drug allergy & 32 \\
confirmed drug anaphylaxis & 16 \\
type I allergy to polyethylene glycol & 19 \\
suspected allergy to polyethylene glycol & 11 \\
confirmed sensitization to polyethylene glycol & 5 \\
type I allergy to polysorbate & \\
suspected allergy to polysorbate & 2 \\
reactions to vaccines & 38 \\
reactions to previous vaccines not further specified & 10 \\
allergic reactions to vaccines & 4 \\
anaphylaxis due to vaccines & 24 \\
contrast medium allergy & 8 \\
confirmed contrast medium allergy & 4 \\
confirmed contrast medium anaphylaxis & 4 \\
type IV sensitizations & 10 \\
type IV (diverse allergens) & 7 \\
confirmed type IV sensitization to PEG & 2 \\
confirmed type IV sensitization to polysorbate & 1 \\
atopy & \\
multiple allergies/ multiple type I sensitizations & 16 \\
atopic dermatitis & 4 \\
asthma & 9 \\
food allergies & 3 \\
venom allergy & 2 \\
other diseases & \\
mast cell disorders & 4 \\
chronic spontaneous urticaria (CSU) & 2 \\
hereditary angioedema (HAE) & 3 \\
autoimmune diseases & 5 \\
cardiovascular diseases & 1 \\
neurological diseases & \\
\hline &
\end{tabular}

*medical history data from $168 / 356$ patients

Table 2: Brighton collaboration level of severity for 162 patients experiencing immediate symptoms in line with a possible allergic reaction after SARS-CoV-2 vaccination 


\begin{tabular}{|c|c|}
\hline Brighton level (number of cases) & Diagnostics \\
\hline $\begin{array}{l}\text { Brighton collaboration level } 1(\mathrm{n}=11) * 3 / 11 \\
\text { revaccinated ( } 1 \text { showed similar symptoms) }\end{array}$ & negative allergy workup $(\mathrm{n}=8)$ \\
\hline $\begin{array}{l}\text { Brighton collaboration level } 2(\mathrm{n}=32) * 12 / 33 \\
\text { revaccinated ( } 1 \text { showed similar symptoms) }\end{array}$ & $\begin{array}{l}\text { positive allergy workup }(n=3) \\
\text { negative allergy workup }(n=24)\end{array}$ \\
\hline $\begin{array}{l}\text { Brighton collaboration level } 3(\mathrm{n}=2) * \text { unknown } \\
\text { status of revaccination }\end{array}$ & $\begin{array}{l}\text { positive allergy workup }(n=8) \\
\text { negative allergy workup }(n=2)\end{array}$ \\
\hline $\begin{array}{l}\text { Brighton collaboration level } 4(\mathrm{n}=10) * 4 / 11 \\
\text { revaccinated ( } 1 \text { showed similar symptoms })\end{array}$ & $\begin{array}{l}\text { positive allergy workup }(\mathrm{n}=0) \\
\text { negative allergy workup }(\mathrm{n}=7)\end{array}$ \\
\hline $\begin{array}{l}\text { Brighton collaboration level } 5(\mathrm{n}=107) * 28 / 108 \\
\text { revaccinated ( } 4 \text { showed similar symptoms })\end{array}$ & $\begin{array}{l}\text { positive allergy workup }(\mathrm{n}=3) \\
\text { negative allergy workup }(\mathrm{n}=91) \\
\text { positive allergy workup }(\mathrm{n}=16)\end{array}$ \\
\hline
\end{tabular}

* re-vaccination status

Table 3: Results of allergy work up of all analysed patients (some patients showed multiple possible results)

\begin{tabular}{|c|c|}
\hline Diagnostic test (SPT,IDT) & Number of patients with positive results \\
\hline SPT Comirnaty $\mathbb{R}$ & 8 \\
\hline IDT Comirnaty@ & $13^{*}$ \\
\hline SPT Vaxzerviaß & 5 \\
\hline IDT Vaxzerviaß & 10 \\
\hline SPT Spikevax@ & 2 \\
\hline IDT Spikevax@ & $9^{*}$ \\
\hline IDT Janssen@R & 1 \\
\hline SPT PEG2000 & 4 \\
\hline IDT PEG2000 & 21 \\
\hline SPT PEG6000 & 2 \\
\hline IDT PEG6000 & 19 \\
\hline SPT PS80 & 8 \\
\hline
\end{tabular}

*2 other patients had systemic reactions after IDT tests (Comirnaty@) and Spikevax@)

SPT:skin-prick-test, IDT: intradermal test, PEG: polyethylene glycol, PS80: polysorbate 80

Table 4: Allergy work up patients meeting the criteria of anaphylaxis according to the Brighton criteria (level 1, 2, 3), $n=46$

\begin{tabular}{ll}
\hline Basic demographics & Number of patients \\
\hline female & $43(93.5 \%)$ \\
median age & $37(21-82)$ \\
Re-vaccination status & Re-vaccination status \\
tolerated re-vaccination & 13 \\
refered after 2nd. vaccination & 3 \\
showed similar tolerability & 2
\end{tabular}




\begin{tabular}{ll}
\hline Basic demographics & Number of patients \\
\hline re-vaccination planned & 2 \\
re-vaccination denied & 2 \\
unknown status & 24 \\
Diagnostic test & Number of patients with positive results \\
SPT Comirnatyß & 1 \\
IDT Comirnatyß & 2 \\
SPT Vaxzervia@ & 1 \\
IDT Vaxzerviaß & 0 \\
SPT Spikevax@ & 0 \\
IDT Spikevax@ & 1 \\
IDT Janssenß & 0 \\
SPT PEG2000 & 1 \\
IDT PEG2000 & 4 \\
SPT PEG6000 & 1 \\
IDT PEG6000 & 2 \\
SPT PS80 & 0 \\
BAT - suspected SARS-Cov-2 vaccine & 3 \\
\hline
\end{tabular}

SPT: skin-prick-test, IDT: intradermal test, BAT: basophil-activation-test

Table 5: Reported suspected anaphylactic reactions from Paul-Ehrlich-Insitute, $n=390$

\begin{tabular}{|c|c|c|c|c|c|c|c|}
\hline Vaccine & 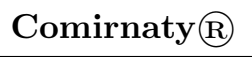 & Comirnaty $\mathbb{R}$ & 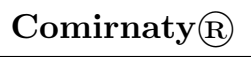 & Spikevax $\AA$ & Spikevax @ & Spikevax @ & Vaxzevria $\AA$ \\
\hline Dose & $1^{\mathrm{st}}$ & $2^{\text {nd }}$ & nk & $1^{\mathrm{st}}$ & $2^{\text {nd }}$ & nk & $1^{\mathrm{st}}$ \\
\hline BC level 1 & 74 & 27 & 1 & 8 & 2 & 0 & 27 \\
\hline BC level 2 & 52 & 19 & 5 & 2 & 1 & 0 & 13 \\
\hline BC level 3 & 8 & 1 & 0 & 1 & 1 & 0 & 0 \\
\hline \multicolumn{8}{|l|}{ Total } \\
\hline BC level 1-3 & 134 & 47 & 6 & 11 & 4 & 0 & 40 \\
\hline BC level 4 & 82 & 21 & 4 & 14 & 0 & 2 & 13 \\
\hline \multicolumn{8}{|l|}{ Total } \\
\hline BC level 1-4 & 216 & 68 & 10 & 25 & 4 & 2 & 53 \\
\hline
\end{tabular}

Nk: not known, BC: Brighton Collaboration 


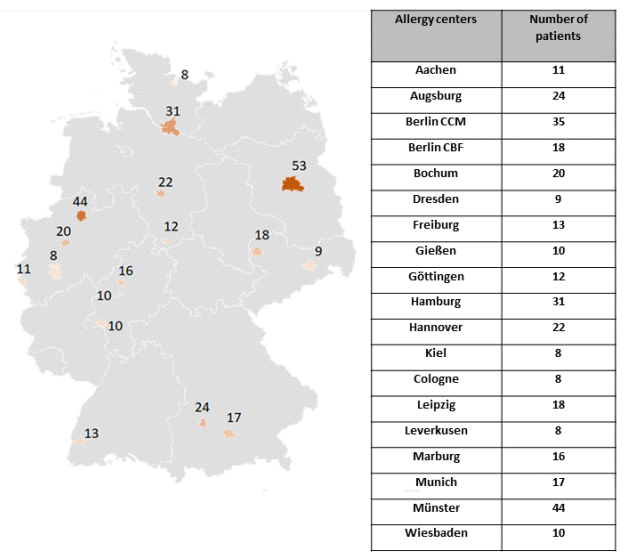

Figure 1 Patients from German allergy centers registered in this analysis

Figure 2: Workflow

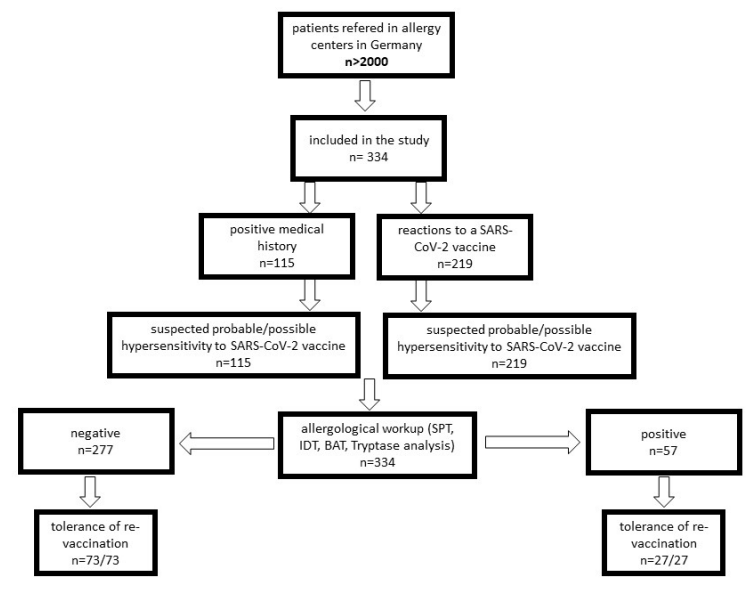

SPT:skin-prick-test, IDT: intradermal test, BAT: basophil-activation-test 